\title{
NOETHERIAN SIMPLE RINGS
}

\author{
BY CARL FAITH \\ Communicated by N. Jacobson, April 20, 1964
}

TheORem 1. A right noetherian simple ring $R$ with identity is isomorphic to the endomorphism ring of a unital torsion-free module $M$ of finite rank over an integral domain.

Since any right artinian ring with identity is right noetherian, this theorem generalizes the classical Wedderburn-Artin theorem which states that a right artinian simple ring with identity is (in the sense of isomorphism) the ring of endomorphisms of a unital module $V$ over a (not necessarily commutative) field $D$.

The implications of this theorem for the structure of $R$ are not yet apparent to the author. For instance, does it imply that $R$ must contain a nontrivial idempotent, if $R$ is not an integral domain?

The conclusion of Theorem 1 holds for any simple ring $R$ with identity which satisfies the maximum conditions on annihilator right ideals and complement right ideals. According to Goldie [2] $R$ will then have a classical right quotient ring $R$ which is a simple artinian ring, that is, a full ring $D_{n}$ of $n \times n$ matrices over a field $D$. Actually, we prove the theorem in the following setting.

THEOREM 2. If $R$ is a simple ring with identity which contains a minimal complement $(=$ closed $=$ uniform) right ideal, then $R$ is the endomorphism ring of a unital torsion-free module over an integral domain.

OUtLINE OF THE PROOF. By a theorem of Utumi [3], the maximal right quotient ring $S$ of $R$ is a full ring of 1.t.'s in a right vector space over a field $D$. It is easily checked that there exists a primitive idempotent $e \in S$ such that $K=e S e \cap R \neq 0$. Then $D=e S e$ is a field, $V=S e$ is a right vector space over $D$, and $S$ is naturally isomorphic to $\Omega=\operatorname{Hom}_{D}(V, V)$ under a map $\phi$ which assigns to each $s \in S$ the element $\phi(s)$ which satisfies $\phi(s) x=s x$, for all $x \in V$. Since $D$ is the right quotient field of $K$ (Faith and Utumi [1]), then $D$ is the right quotient field of the subring $\Delta$ generated by $K$ and $e$. Furthermore, $M=S e \cap R$ is a unital torsion-free module over $\Delta$ and it can be shown that $V=M D=\left\{x d^{-1} \mid x \in M, 0 \neq d \in \Delta\right\}$. Therefore any element $\gamma$ in $\Gamma=\operatorname{Hom}_{\Delta}(M, M)$ has a unique extension $\gamma^{\prime}$ in $\Omega$. The natural isomorphism $S \cong \Omega$ implies that $\Gamma$ is isomorphic to the subring $T=\{s \in S \mid s M \subseteq M\}$. Since $T$ contains $R$, in order to establish 
$R \cong \operatorname{Hom}_{\Delta}(M, M)$, it remains only to show that $T \subseteq R$. Simplicity of $R$ implies $M R=R$, and then

$$
R=M R=(T M) R=T(M R)=T R \supseteq T,
$$

that is, $T \subseteq R$.

When $S$ is the classical quotient ring of $R$, then Goldie's theorems imply that $V$ is finite dimensional over $D$, and then $M$ will have finite rank over $\Delta$.

We state the following corollaries without comment.

COROLlary 3. Let $R$ be a simple ring with identity containing an idempotent $e \neq 0$, such that $\Delta=e R e$ is an integral domain. Then if $\Delta$ has a right quotient field, e.g., if $R$ is right noetherian, then $R$ $\cong \operatorname{Hom}_{\Delta}(R e, R e)$. $R$.

$R$ is a right order in $S$ in case $S$ is a classical right quotient ring of

CoRollary 4. Let $S=\operatorname{Hom}_{D}(V, V)$, where $V$ is a right vector space over $D$, and let $R$ be a right order in $S$. Then, if either $R$ is a simple ring with identity, or if $R$ is a maximal right order in $S$, then there exist a right order $\Delta$ of $D$ containing an identity, and a $\Delta$-submodule $M$ of $V$ such that $R$ is naturally isomorphic to $\operatorname{Hom}_{\Delta}(M, M)$.

\section{REFERENCES}

1. Carl Faith and Yuzo Utumi, On noetherian prime rings, Trans. Amer. Math. Soc. (1964) (to appear).

2. A. W. Goldie, Semiprime rings with maximum condition, Proc. London Math. Soc. (3) 10 (1960), 201-220.

3. Yuzo Utumi, On quotient rings, Osaka Math. J. 8 (1956), 1-18.

Rutgers, The State University 